Proceeding Series of the Brazilian Society of Computational and Applied Mathematics

\title{
Utilização da nova metaheurística Alcateia na identificação de danos estruturais
}

\section{Cleber de Almeida Corrêa Junior ${ }^{1}$ Rosilene Abreu Portella Corrêa ${ }^{2}$}

Instituto do Noroeste Fluminense de Educação Superior, INFES, Universidade Federal Fluminense, UFF, Santo Antônio de Pádua, RJ

Isabela Cristina da Silveira e Silva Rangel ${ }^{3}$

Luciano dos Santos Rangel ${ }^{4}$

Leonardo Tavares Stutz ${ }^{5}$

Instituto Politécnico do Estado do Rio de Janeiro, IPRJ, Universidade do Estado do Rio de Janeiro, UERJ, Nova Friburgo, RJ

Resumo. O presente trabalho propõe um novo método estocástico de otimização global denominado Alcateia, o qual é um método baseado no comportamento dos lobos. Suas principais características é a necessidade de apenas um parâmetro de controle a ser calibrado e fácil implementação. Análises numéricas considerando a identificação de danos estruturais em uma estrutura do tipo viga de Euler-Bernoulli simplesmente apoiada utilizando os dados baseados no domínio da frequência foi realizada, com intuito de verificar o desempenho do método proposto. Diante dos resultados apresentados, pode-se considerar que o método conseguiu localizar e quantificar corretamente os danos na estrutura, mesmo considerando ruído aditivo.

Palavras-chave. Algoritmo Alcateia, Identificação de danos estruturais, Frequências naturais.

\section{Introdução}

Uma estrutura, durante seu tempo útil, está sujeita aos mais diversos processos de deterioração, sejam estes provocados pelo homem ou pela própria natureza, como ventos, maresia, entre outros, colocando sua integridade estrutural em risco, sendo assim, é de

\footnotetext{
${ }^{1}$ cleberacj@yahoo.com.br

${ }^{2}$ rosiportella@yahoo.com.br

3 isabelacssilva@yahoo.com.br

${ }^{4}$ lucianosrangel@yahoo.com.br

${ }^{5}$ ltstutz@iprj.uerj.br
} 
extrema importância o monitoramento contínuo da estrutura e identificação de danos em um estágio inicial.

Os métodos de identificação de danos geralmente são baseados em: dados modais (análise modal), dados no domínio do tempo e dados no domínio da frequência [2]. O presente trabalho considera parâmetros modais da estrutura, especificamente, as frequências naturais não amortecidas.

Um tema de bastante importância quando se deseja identificar danos estruturais, refere-se ao método de otimização a utilizar. Os métodos determinísticos, apesar de seu extenso uso, sofrem ainda algumas limitações como problemas de divergência e instabilidade nos cálculos numéricos e são susceptíveis a ficarem presos em pontos ótimos locais. Autores têm aplicados métodos de otimização classificados como estocásticos devido à necessidade de lidar com particularidades dos problemas de identificação de danos com a presença de ruído.

Dentre alguns trabalhos, podemos citar o algoritmo Evolução Diferencial (ED) aplicado na identificação de danos estruturais a partir de sua resposta impulsiva [5], onde obteve resultados satisfatórios. É realizada uma comparação entre os métodos de otimização Simulated Annealing (SA) e Particle Swarm Optimization (PSO) em um problema de identificação de danos em [1]. Melhores resultados foram encontrados utilizando-se o SA, porém segundo o autor, seu elevado custo computacional torna-se um grave fator. Quanto ao PSO, relata-se a dificuldade de se obter uma configuração ótima de seus parâmetros.

No presente trabalho, é apresentado um novo método estocástico denominado Alcateia, o qual é um método de otimização global, baseado no comportamento dos lobos, onde este, apresenta como característica a necessidade de apenas um parâmetro de controle a ser calibrado e fácil implementação. Com intuito de validar o método, foi realizada a identificação de danos estruturais em uma estrutura do tipo viga de Euler-Bernoulli simplesmente apoiada, apresentando resultados com elevada acurácia.

\section{Modelagem do problema de identificação de danos}

$\mathrm{Na}$ estratégia de identificação de danos adotada, a integridade da estrutura é considerada como sendo continuamente descrita, no domínio do corpo, por um parâmetro estrutural denominado parâmetro nodal de coesão ( $\beta)$ [6]. Este parâmetro está relacionado com a ligação entre os pontos materiais e pode ser interpretado como uma medida do estado de coesão local do material, onde $0 \leq \beta \leq 1$.

Neste trabalho, foi considerado que o dano afeta apenas as propriedades elásticas da estrutura, hipótese comumente adotada na literatura. Deste modo, a matriz de rigidez do Modelo de Elementos Finitos (MEF) da estrutura pode ser escrita como

$$
\mathbf{K}\left(\beta_{h}\right)=\int_{\Omega} \beta(x) E_{0} I_{0} \mathbf{H}^{T}(x) \mathbf{H}(x) d \Omega,
$$

onde $\mathbf{H}$ é o operador diferencial discretizado, $E_{0}$ e $I_{0}$ são, respectivamente, os valores nominais do módulo de elasticidade e do momento de inércia de área e $\beta$ representa o campo de coesão no domínio elástico $\Omega$ da estrutura. Considerando uma viga de seção 
transversal retangular e com módulo de elasticidade uniforme, o campo de coesão pode ser escrito como

$$
\beta(x)=\left(h(x) / h_{0}\right)^{3},
$$

onde $h_{0}$ e $h(x)$, indicam, respectivamente, a espessura nominal e a espessura da viga na posição $x$. Portanto, nos nós defeituosos tem-se $h(x) / h_{0}<1$, e nos nós onde não há danos, tem-se $h(x) / h_{0}=1$. No presente trabalho, as frequências naturais não-amortecidas serão utilizadas, sendo estas, obtidas a partir do seguinte problema de autovalor-autovetor generalizado, escrito na forma matricial como

$$
\mathbf{K} \Phi=\Lambda \mathrm{M} \Phi
$$

onde $\mathbf{M}$ é a matriz de massa e as matrizes modais $\boldsymbol{\Phi}$ e $\boldsymbol{\Lambda}$ constituem, respectivamente, a matriz de autovetores e autovalores, dadas por

$$
\boldsymbol{\Phi}=\left[\begin{array}{llll}
\boldsymbol{\Phi}_{1} & \mathbf{\Phi}_{2} & \ldots & \boldsymbol{\Phi}_{n}
\end{array}\right] \quad \boldsymbol{\Lambda}=\operatorname{diag}\left(\omega_{i}^{2}\right), \quad i=1, \ldots, n,
$$

onde $\boldsymbol{\Phi}_{i}$ representa a i-ésima forma modal da estrutura e $\omega_{i}^{2}$ sua correspondente frequência natural não-amortecida.

A literatura indica a utilização de técnicas de identificação de danos estruturais e monitoramento de estruturas fundamentadas no ajuste de um MEF. Mas, geralmente obtêm-se de um problema mal posto e um elevado custo computacional, inerente a essas técnicas. Para contornar esse aspecto, pode-se utilizar o Modelo de Superfície de Resposta (MSR) em substituição a um MEF da estrutura, onde este, apresenta como vantagem a redução do custo computacional para a solução de problemas inversos de identificação de danos [3].

\subsection{Modelo de Superfície de Resposta}

O MSR é obtido através das relações entre parâmetros da estrutura e respostas de interesse. Desta forma, para uma dada resposta escalar y, tem-se

$$
y=f\left(\beta_{1}, \beta_{2}, \ldots, \beta_{n p}\right)+\varepsilon,
$$

onde $f\left(\beta_{1}, \beta_{2}, \ldots, \beta_{n p}\right)$ representa a relação entre a resposta e as variáveis independentes e $\varepsilon$ sendo o resíduo. Em geral, os parâmetros do modelo devem ser codificados, em $x_{i} \in[-1,1]$ [4]. Esta relação pode ser aproximada por polinômios de baixa ordem em algumas regiões relativamente pequenas do espaço definido pelas variáveis independentes, sendo então comumente utilizados modelos de primeira ou segunda ordem. Dessa forma, a Eq. (5) pode ser reescrita como

$$
\hat{y}=f\left(x_{1}, x_{2}, \ldots, x_{n p}\right)+\varepsilon=\hat{b}_{0}+\sum_{i=1}^{n p} \hat{b}_{i} x_{i}+\sum_{i=1}^{n p} \hat{b}_{i i} x_{i}^{2}+\sum_{i<j}^{n p} \sum_{j=2}^{n p} \hat{b}_{i j} x_{i} x_{j} .
$$

No presente trabalho, a identificação de danos estruturais considera o ajuste de um MSR da estrutura, objetivando-se a minimização de uma função de erro definida a partir 
das frequências naturais experimentais $\left(\mathbf{v}_{E}\right)$ e das correspondentes frequências previstas pelos $\operatorname{MSR}(\hat{\mathbf{v}}(\boldsymbol{\beta}))$ e $\mathcal{W}$ é uma matriz de ponderação

$$
\min _{\boldsymbol{\beta}} \mathcal{F}=\min _{\boldsymbol{\beta}}\left(\left(\mathbf{v}_{E}-\hat{\mathbf{v}}(\boldsymbol{\beta})\right)^{T} \mathcal{W}\left(\mathbf{v}_{E}-\hat{\mathbf{v}}(\boldsymbol{\beta})\right)\right)
$$

\section{Método estocástico Alcateia}

Os lobos, para conseguirem ter sucesso em suas caçadas e para estarem mais protegidos, geralmente vivem em conjunto com outros lobos, caracterizando assim, uma alcateia. As alcateias são grupos organizados hierarquicamente e no nível mais alto, encontra-se o lobo alfa, podendo ser macho ou fêmea, pois as posições de importância dos lobos não se baseiam no sexo, mas em força e autoridade. O lobo alfa é responsável por observar as melhores presas, ordenar as caçadas, delimitar territórios e proteger seus subordinados.

Em um nível mais abaixo, encontra-se o lobo beta, que exerce a função de um segundo comandante (na ausência do lobo alfa) e assumindo a liderança da alcateia caso o lobo alfa venha a falecer. Como as posições dos lobos são baseadas na força e autoridade, lobos betas podem desafiar o lobo alfa, em situações onde o lobo alfa encontra-se muito velho ou doente. Por último, encontra-se o lobo ômega, o mais fraco e mais submisso na alcateia.

\subsection{Algoritmo Alcateia}

O algoritmo Alcateia requer de poucos parâmetros de inicialização, sendo eles, o raio de busca inicial $(r)$ (região viável pela busca do ótimo global), limites das coordenadas dos lobos da alcateia, número de iterações externas $\left(n_{e}\right)$ e internas $\left(n_{i}\right)$ e apenas um parâmetro precisa ser calibrado, o parâmetro de independência $(i d)$ dos lobos.

As iterações internas são utilizadas para realização da atualização de cada lobo e posteriormente, a seleção dos melhores lobos. As iterações externas são utilizadas para a redefinição da hierarquia da alcateia, ou seja, após o processo de seleção dos novos lobos, é necessário estabelecer o novo líder da alcateia, o lobo alfa $\left(l_{a}\right)$. Os lobos restantes serão atraídos (ou dispersos) para o lobo alfa, segundo o parâmetro de independência, um número real pertencente ao intervalo $[0,1]$, fornecido pelo usuário. Por último, é realizada a contração do raio de busca.

Seja uma alcateia inicial, comumente gerada por uma distribuição de probabilidade uniforme, constituída por $N_{l}$ lobos (com $n_{l}$ componentes representando cada variável do projeto), percorrendo todo o espaço de busca e mantendo-se fixa a quantidade de lobos durante todo o processo de minimização.

A atualização da alcateia inicial da iteração é obtida gerando perturbações aleatórias no raio de busca e realizando uma soma vetorial entre cada lobo da alcateia inicial com as perturbações aleatórias. O processo de atualização pode ser expresso pela Eq. (8):

$$
l_{i+1}=l_{i}+r *\left(-0.5+\operatorname{rand}_{i}()\right)
$$

com $\mathrm{i}=1, \ldots, N_{l}$ e $\operatorname{rand}_{i}$ um número gerado aleatoriamente no intervalo $[0,1]$. 
Após o processo de atualização, passa-se para o processo de seleção dos melhores lobos. A seleção acontecerá em relação ao valor do funcional de cada lobo, realizando a comparação entre o lobo da alcateia inicial da iteração $\left(l_{i}\right)$ com o lobo atualizado $\left(l_{i+1}\right)$. Se o lobo $\left(l_{i+1}\right)$ ocasionar na redução do valor da função objetivo, este tomará o lugar do antigo lobo $\left(l_{i}\right)$, caso contrário, não haverá substituição na alcateia.

Terminada a atualização e seleção, passa-se para a fase de redefinição da hierarquia, para isso, é necessário encontrar o lobo da nova alcateia que gere o menor valor na função objetivo, caracterizando assim, o lobo alfa.

O parâmetro id indica o grau de independência dos componentes da alcateia. Quanto mais próximo de zero, maior será a dependência dos lobos, consequentemente, todos os lobos irão se aproximar do lobo alfa, caso o parâmetro $i d$ tenha um valor próximo de um, os lobos terão maior autonomia e consequentemente, poderão se afastar do lobo alfa. O processo de aproximação (ou dispersão) pode ser observado pela Eq. (9). Por último, deve-se reduzir o espaço de busca, conforme a Eq (10).

$$
\begin{gathered}
l=l * i d+(1-i d) * l_{a} \\
r_{i}=l_{i}-l_{i-1}
\end{gathered}
$$

Esses processos são repetidos até que algum critério de parada seja alcançado.

\section{Resultados Numéricos}

Utilizou-se uma viga de aço simplesmente apoiada com 1,46 m de comprimento, 7, $93 \times$ $10^{-3} \mathrm{~m}$ de espessura, $7,62 \times 10^{-2} \mathrm{~m}$ de largura, momento de inércia de área $3,17 \times 10^{-9}$ $\mathrm{m}^{4}$, módulo de elasticidade $2,07 \times 10^{11} \mathrm{~Pa}$ e massa específica $7,85 \times 10^{3} \mathrm{~kg} / \mathrm{m}^{3}$.

A viga em questão foi discretizada pelo MEF em 24 elementos bidimensionais do tipo Euler-Bernoulli, onde cada elemento apresenta dois nós e cada nó possui dois graus de liberdade (GDL) (rotação e translação) e um parâmetro de coesão. Portanto, a estrutura possui 25 parâmetros nodais de coesão e, devido às condições de contorno, 48 GDL.

No entanto, a partir de uma análise do comportamento da viga em relação às suas frequências naturais, observou que danos de mesma magnitude e em posições simétricas resultam nas mesmas variações nas frequências naturais [4]. Com isso, a viga foi considerada como sendo formada por subestruturas, diminuindo o número de parâmetros nodais de coesão a serem utilizados na geração das superfícies de resposta. As subestruturas são definidas de forma indireta, através de parâmetros de coesão simétricos, vide Figura 1.

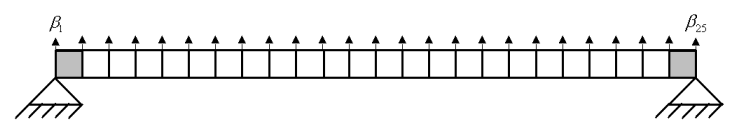

Subestrutura 1

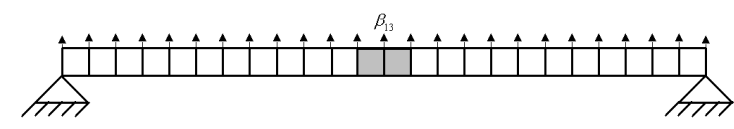

Subestrutura 13

Figura 1: Divisão das subestruturas presentes na viga simplesmente apoiada.

A Tabela 1, apresenta os cenários de danos que serão considerados neste trabalho. 
Tabela 1: Cenários de danos

\begin{tabular}{|c|c|c|c|c|}
\hline Caso & Subestrutura & Posição $(\mathrm{m})$ & $h(x) / h_{0}$ & Nível de ruído (\%) \\
\hline 1 & 6 & 0,3041 & 0,8 & 0 \\
\hline 2 & 13 & 0,730 & 0,8 & 0 \\
\hline 3 & $6 ; 13$ & 0,$3041 ; 0,730$ & 0,$8 ; 0,8$ & 1 \\
\hline
\end{tabular}

Em todos os casos considerados, o dano foi definido por uma redução de $20 \%$ na altura relativa da seção transversal da viga em $x=0,3041 \mathrm{~m}, x=0,730 \mathrm{~m}$, para os Casos $1 \mathrm{e}$ 2 , respectivamente, e as frequências naturais não estão corrompidas por ruído. No Caso 3 foi considerado um dano duplo nas posições dos Casos 1 e 2, e as frequências naturais foram corrompidas com $1 \%$ de ruído.

Devido à aleatoriedade dos métodos estocásticos, foram realizadas 10 simulações com o método Alcateia e o resultado final apresentado foi obtido da média aritmética envolvendo os resultados parciais. Para a inicialização do método, foram utilizados os seguintes valores: tamanho da Alcateia $N_{l}=120$, raio de busca $r=\left[\begin{array}{ll}-0,1 & 0,1\end{array}\right]$, número de iterações internas $n_{i}=1000$, número máximo de iterações externas $n_{i}=50$ e coeficiente de independência $i d=0,3$. O critério de parada adotado foi o número máximo de iterações externas ou a tolerância do funcional de $10^{-5}$.

A Figura 2 apresenta a média das dez simulações utilizando o método estocástico Alcateia para os três cenários de danos apresentados.

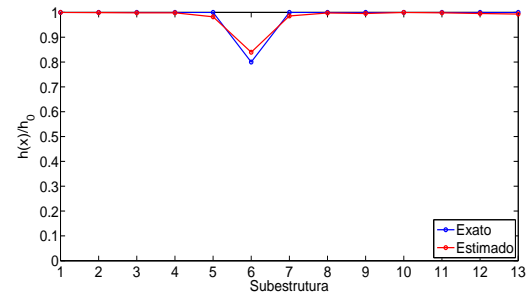

(a) Caso 1

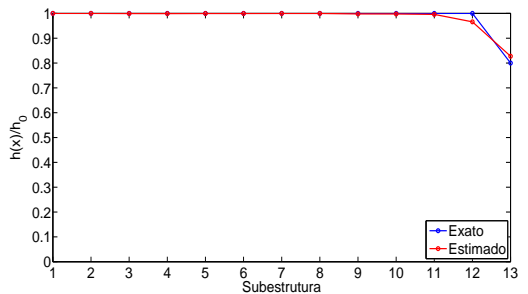

(b) Caso 2

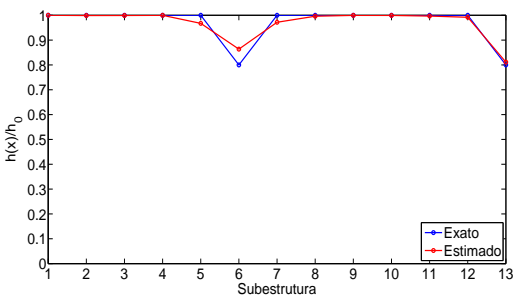

(c) Caso 3

Figura 2: Identificação de danos utilizando o método Alcateia.

Pela Figura 2, é possível perceber que o algoritmo conseguiu localizar corretamente as subestruturas danificadas em todos os casos. Nos Casos 1 e 2, observa-se que a abordagem proposta foi capaz de identificar com acurácia a localização do dano. Porém no Caso 3, indicou uma intensidade ligeiramente menor do dano na subestrutura 6 , visto que este caso está corrompido com $1 \%$ de ruído. 


\section{Conclusão}

No presente trabalho, um MSR ajustado para as frequências naturais foi adotado para a identificação de danos estruturais em uma viga de Euler-Bernoulli simplesmente apoiada. A partir dos resultados obtidos, observou-se que o método estocástico Alcateia foi capaz de localizar e quantificar as subestruturas danificadas com elevada acurácia, mesmo considerando ruído aditivo. Como sugestões para trabalhos futuros, a aplicação do algoritmo Alcateia em estruturas do tipo viga, com outras condições de contorno, estruturas do tipo placa e em sistemas de equações não lineares.

\section{Agradecimentos}

Os autores deixam aqui registrados os agradecimentos às instituições INFES/UFF e IPRJ/UERJ pelo apoio concedido.

\section{Referências}

[1] O. Bengambre e J. E. Laier, A hybrid Particle Swarm Optimization - Simplex algorithm (PSOS) for structural damage identification. Advances in Engineering Software. vol. 40, pp. 883-891, (2009).

[2] R. A. P. Corrêa, Identificação de danos em estruturas bi-dimensionais via matriz de flexibilidade baseada em um modelo de dano contínuo. 129 f. Tese de Doutorado em Modelagem Computacional, Instituto Politécnico, Universidade do Estado do Rio de Janeiro, Nova Friburgo, (2013).

[3] S. E. Fang e R. Perera, A response surface methodology based damage identification technique. J. Iop Poblishing, vol. 18, pp. 14,( 2009).

[4] I. C. S. S. Rangel, Identificação de danos estruturais a partir do modelo de superfície de resposta. 109 f. Dissertação de Mestrado em Modelagem Computacional, Instituto Politécnico, Universidade do Estado do Rio de Janeiro, Nova Friburgo, (2014).

[5] L. S. Rangel, Identificação de danos estruturais utilizando dados no domínio do tempo provenientes de esaios de vibração. 106 f. Dissertação de Mestrado em Modelagem Computacional, Instituto Politécnico, Universidade do Estado do Rio de Janeiro, Nova Friburgo, (2014).

[6] L. T. Stutz, D. A. Castello, F. A. Rochinha, A flexibility-based continuum damage identification approach. Journal of Sound and Vibration, 279, pp. 641-667, (2005). 